\title{
Enantioselective synthesis of strobamine and its analogues
}

\author{
Xing Zhang, ${ }^{\text {a }}$ Philip Abraham, ${ }^{\text {a }}$ S. Wayne Mascarella, ${ }^{a}$ Judith Flippen-Anderson, \\ Jeffrey R. Deschamps, ${ }^{b}$ and F. Ivy Carroll ${ }^{a^{*}}$
}

${ }^{a}$ Center for Organic and Medicinal Chemistry, Research Triangle Institute, P O Box 12194, Research Triangle Park, North Carolina 27709-2194, USA and ${ }^{b}$ Naval Research Laboratory, Code 6930, 4555 Overlook Avenue, Washington, DC 20375, USA

E-mail: fic@rti.org

\section{Dedicated to Professor James M. Cook, celebrating his $65^{\text {th }}$ birthday}

\begin{abstract}
$(2 S, 5 S, 8 R)$-Strobamine $(+)-\mathbf{1 a}$ was synthesized by adding cinnamoyl cyanide $\mathbf{6 a}$ to tropinone $\mathbf{3}$ lithium enolate generated by treatment of $\left(S, S^{\prime}\right)$ - $\alpha, \alpha$-dimethyldibenzylamide with butyl lithium in the presence of lithium chloride to give (-)-chalcostrobamine (-)-7a, which yielded a one-to-one mixture of $(+)-1 a$ and its $\mathrm{C}-2$ epimer $(-)$-2a on treatment with $2 \mathrm{~N}$ sulfuric acid. Compounds $(+)-$ 1a and (-)-2a could be separated by column chromatography. (-)-Strobamine (-)-1a and (+)-2a were synthesized by a similar set of reactions using the tropinone 3 lithium enolate generated with butyl lithium in the presence of $\left(R, R^{\prime}\right)-\alpha, \alpha^{\prime}$-dimethyldibenzylamide and lithium chloride. $(+)$ - and (-)-p-Methylstrobamine (+)- and (-)-1b and (+)- and (-)-epi-p-methylstrobamine $(+)-$ and (-)-2b were synthesized by a similar procedure. The absolute configuration of (+)-epi-pmethylstrobamine $(+)$-2b was determined by X-ray analysis to have the $(2 S, 5 R, 8 S)$ configuration.
\end{abstract}

Keywords: Strobamine, epi-strobamine, heterocycles

\section{Introduction}

Strobamine (+)-1a was first isolated from the leaves of the endemic New Caledonian plant Knightia strobilina Labill in 1980. ${ }^{1,2}$ However, the synthesis of $(+)-\mathbf{1 a}$ has not been reported, and its absolute stereochemistry is unknown. The synthesis of $( \pm)$-strobamine $( \pm)$-1a and $( \pm)$-epistrobamine $( \pm)$-2a was reported by Lounasmaa et al. in $1983 .^{3}$ In their preparation, tropinone was deprotonated with $\mathrm{NaH}$, and the resulting enolate was treated with cinnamoyl cyanide to form chalcostrobamine $( \pm)$-7a. Subsequent cyclization under acidic conditions afforded $( \pm)$-1a and $( \pm)$-2a. To the best of our knowledge, no additional report has been published on the synthesis or the absolute configuration of strobamine. The observation that $( \pm)$-strobamine, prepared in our 
laboratory, showed an $\mathrm{IC}_{50}$ of $370 \mathrm{nM}$ for inhibition of radioligand binding at the dopamine transporter, suggested that it would be interesting to evaluate the $(+)$ - and (-)-isomers of strobamine $(+)$ - and (-)-1a as well as its epimers $(+)-$ and $(-)-\mathbf{2 a}$. In this communication, we report the enantioselective synthesis of $(+)$ - and (-)-1a and their epimers $(+)-$ and $(-)-2 a$. In addition, we report the synthesis of $(+)-$ and $(-)$-p-methylstrobamine $(+)$ - and (-)-1b, $(-)$-epi-pmethylstrobamine $(+)$ - and $(-)-\mathbf{2 b}$, and the X-ray structure of $(+)-\mathbf{2 b}$.

\section{Results and Discussion}

Since Majewski and Lazny ${ }^{4}$ reported that (-)-chalcostrobamine (-)-7a and (+)-entchalcostrobamine $(+)-7$ a could be synthesized in $>95 \%$ ee by enantioselective deprotonation of tropanone 3 using chiral lithium $\left(S, S^{\prime}\right)$ - $\alpha, \alpha^{\prime}$-dimethylbenzylamide 4 and $\left(R, R^{\prime}\right)-\alpha, \alpha^{\prime}-$ dimethyldibenzylamide 5, we envisioned that $(+)$ - and $(-)-\mathbf{1 a},(+)-$ and $(-)-\mathbf{2 a}$ as well as $(+)-\mathbf{1 b}$ and $(-)-\mathbf{1 b}$ and $(-)-\mathbf{2 b}$ and $(+)-\mathbf{2 b}$ could be prepared by the route shown in Scheme 1. We first repeated the synthesis reported by Majewski and Lazny ${ }^{4}$ and, as expected, we found that the use of chiral amides 4 and 5 led to $(-)-7 \mathbf{a}$ and $(+)-7 \mathbf{a}$, respectively.

With the structures of $(+)$ - and $(-)$-7a verified, strobamine $(+)-\mathbf{1 a}$ and its analogues were synthesized from tropinone 3 in a one-pot reaction. Lithium $\left(R, R^{\prime}\right)-\alpha, \alpha^{\prime}$-dimethyldibenzylamide 4 and lithium $\left(S, S^{\prime}\right)$ - $\alpha, \alpha^{\prime}$-dimethyldibenzylamide 5 were generated in situ from the corresponding amine hydrochloride and 1.98 equivalents of $n$-BuLi. The required tropinone lithium enolates were then produced by adding the lithium amide base to a tetrahydrofuran solution of tropinone 3. Treatment of the above enolates derived from 4 or 5 with aroyl cyanides ${ }^{5} \mathbf{6 a}$ or $\mathbf{6 b}$ afforded $(+)$ - or (-)-chalcostrobamines 7a or 7b, respectively (Scheme 1). (-)-Chalcostrobamines (-)-7a or $(-)$-7b were cyclized under acidic $\left(\mathrm{H}_{2} \mathrm{SO}_{4}\right)$ conditions to give nearly equal quantities of $(+)-\mathbf{1 a}$ or $(+)-\mathbf{1 b}$ and their $\mathrm{C}-2$ epimers $(-)-\mathbf{2 a}$ or $(-)-\mathbf{2} \mathbf{b}$, which were separated by chromatography. Cyclization of $(+)-\mathbf{7 a}$ or $(+)-\mathbf{7 b}$ under the same conditions provided $(-)-\mathbf{1 a}$ or $(-)-\mathbf{1 b}$ and $(+)-\mathbf{2 a}$ or $(+)-\mathbf{2 b}$. The absolute configuration of $(2 S, 5 R, 8 S)-(+)-e p i-p$-methylstrobamine $(+)-2 \mathbf{b}$ was assigned on the basis of X-ray spectroscopic analysis (Figure 1). The NMR spectra and optical rotation of $(+)$-strobamine synthesized in this study were consistent with natural $( \pm)$-strobamine; see comparison of the ${ }^{13} \mathrm{C}$ NMR data in Table 1; for additional details, see the Experimental Section. Major differences in the ${ }^{13} \mathrm{C}$ NMR spectra are the lower field chemical shifts of C-2, C3, and C-7 and higher field shifts for C-6 and C-9 for strobamine 1a relative to epi-strobamine 2a (Table 1). Because (+)-2b was shown by X-ray analysis to have the $(2 S, 5 R, 8 S)$ configuration, ()-strobamine (-)-1a, which was generated in the same type of reaction, has the $(2 \mathrm{R}, 5 \mathrm{R}, 8 \mathrm{~S})$ configuration and its enantiomer, $(+)$-strobamine $[(+)-1 \mathbf{a}]$, has the $(2 S, 5 S, 8 R)$ configuration.

The $\mathrm{IC}_{50}$ value of $265 \mathrm{nM}$ for the inhibition of binding to the dopamine transporter for $(+)-\mathbf{1 a}$ was not appreciably different from that of the racemate. 


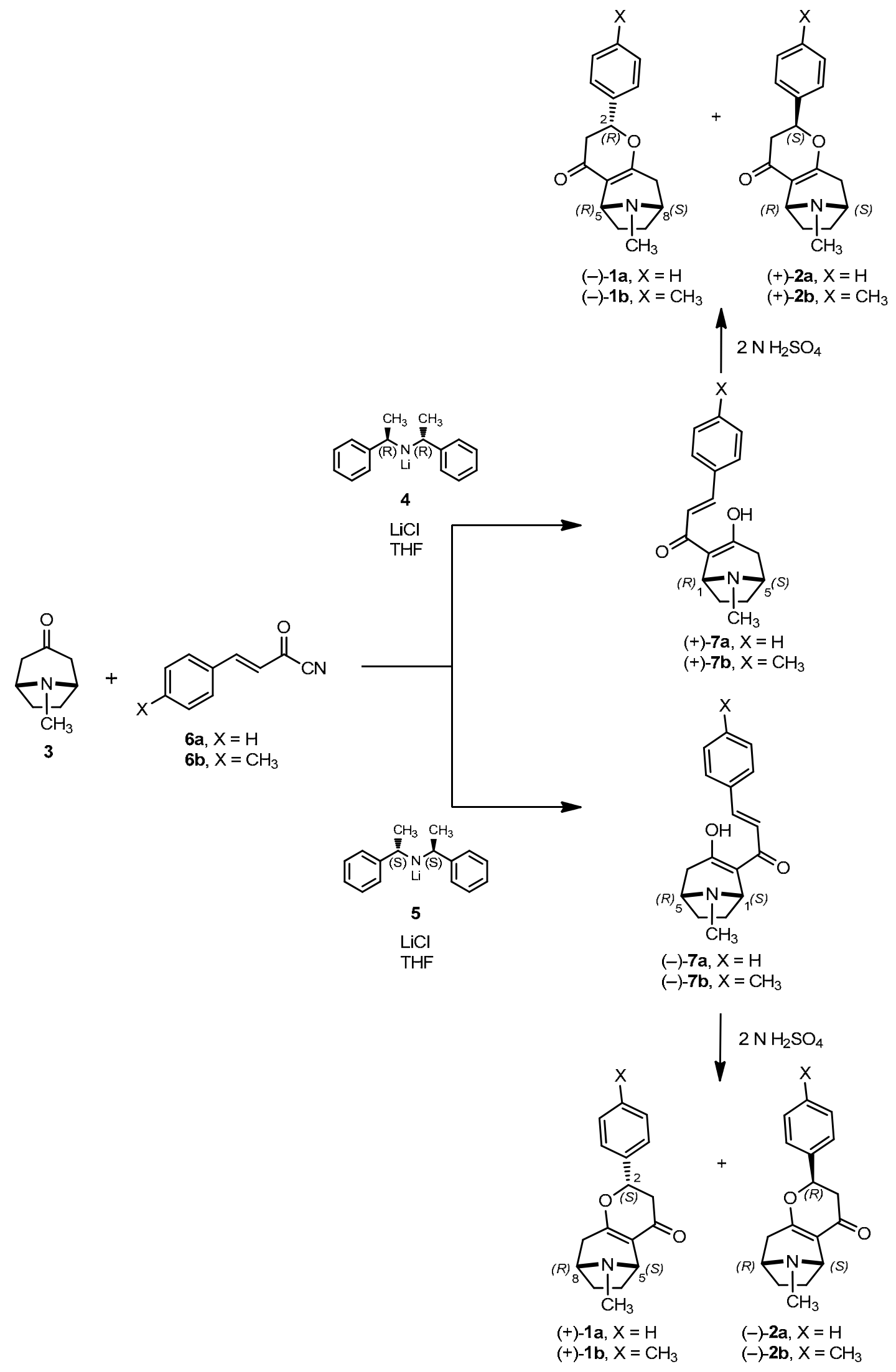

Scheme 1 


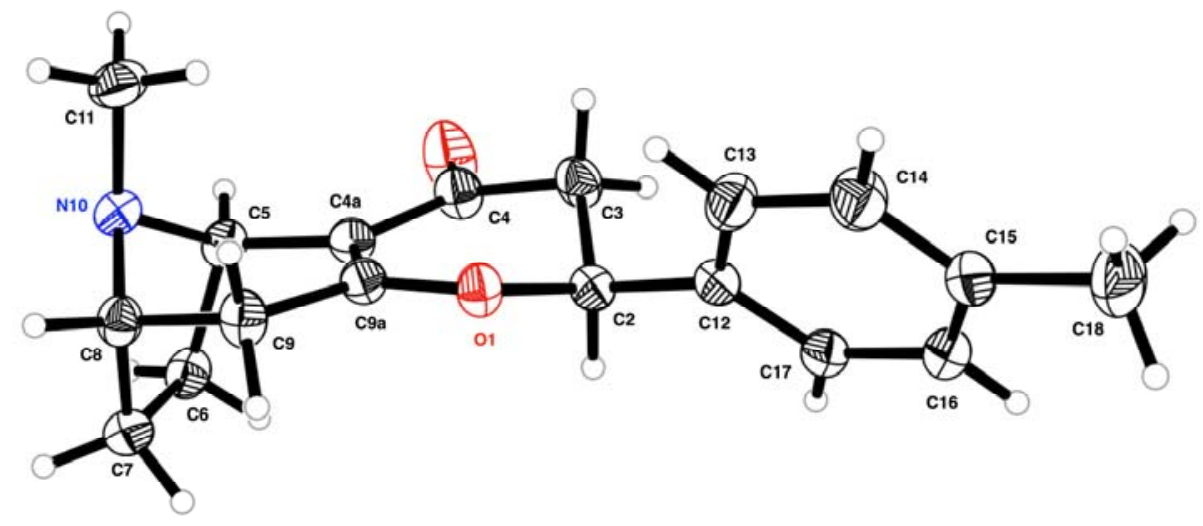

Figure 1. Structure of compound (+)-2b showing labeling of the non-hydrogen atoms.

Table 1. Comparison of ${ }^{13} \mathrm{C}$ NMR data for (+)-strobamine (+)-1a and (-)-epi-strobamine (-)-2 to reported values for $( \pm)$-strobamine and $( \pm)$-epi-strobamine ${ }^{\mathrm{a}}$

\begin{tabular}{ccccc}
\hline $\mathrm{C}$ & $(+) \mathbf{- 1 a}$ & $(-)-2 \mathbf{a}$ & $( \pm)-\mathbf{1} \mathbf{a}^{\mathrm{a}}$ & $( \pm)-\mathbf{2} \mathbf{a}^{\mathrm{a}}$ \\
& \multicolumn{1}{c}{$\delta_{\mathrm{c}}$} & \multicolumn{1}{c}{$\delta_{\mathrm{c}}$} & \multicolumn{1}{c}{$\boldsymbol{\delta}_{\mathrm{c}}$} & \multicolumn{1}{c}{$\delta_{\mathrm{c}}$} \\
\hline 2 & 80.80 & 79.84 & 80.5 & 79.7 \\
3 & 42.90 & 42.33 & 42.7 & 42.2 \\
4 & 189.63 & 189.44 & 189.2 & 189.2 \\
$4 \mathrm{a}$ & 117.19 & 116.73 & 116.8 & 116.4 \\
5 & 58.25 & 58.22 & 58.0 & 58.0 \\
7 & 35.65 & 34.74 & 35.4 & 34.6 \\
6 & 32.70 & 33.46 & 32.6 & 33.3 \\
8 & 55.93 & 55.18 & 55.7 & 55.0 \\
9 & 28.45 & 29.16 & 28.3 & 29.0 \\
$9 \mathrm{a}$ & 168.94 & 168.82 & 168.6 & 168.6 \\
10 & 37.64 & 36.95 & 37.4 & 36.8 \\
\hline
\end{tabular}

${ }^{\mathrm{a}}$ Values taken from ref 3.

\section{Conclusions}

The enantioselective synthesis of the natural product $(+)$-strobamine $(+)-\mathbf{1 a}$ and its $p$-methyl analogue has been achieved. The absolute configuration of strobamine has also been determined for the first time by NMR and X-ray spectroscopic analysis of the (+)-p-methyl analogue (+)-2b. 


\section{Experimental Section}

General. ${ }^{1} \mathrm{H}$ and ${ }^{13} \mathrm{C}$ NMR spectra were recorded on a Bruker 300 Spectrometer using TMS as an internal standard. Optical rotations were measured with an Autopol IV automatic polarimeter. HPLC analyses were carried out on a Dynamax HPLC system. The optical purity was determined by chiral HPLC [Sumichiral OA-4900 $(4.6 \mathrm{~mm} \times 25 \mathrm{~cm})$. Flash column chromatography was performed using EM Science silica gel 60 (particle size 40-63 $\mu \mathrm{m}$ ). CMA80 is a mixture of $80 \%$ chloroform, $18 \%$ methanol, and $2 \%$ concentrated ammonium hydroxide. Elemental analyses were performed by Atlantic Microlab, Inc., Atlanta, GA.

\section{General procedure for the synthesis of chalcostrobamine (7a) and (7b)}

Using a procedure similar to that reported by Majewski and Lazny, ${ }^{4}$ a solution of $n$-BuLi (0.95 $\mathrm{mL}, 2.5 \mathrm{M}, 2.4 \mathrm{mmol})$ in hexane was added dropwise to a solution of bis[(R,R)- or $(S, S)$ phenylethylamine $] \cdot \mathrm{HCl}(0.30 \mathrm{~g}, 1.2 \mathrm{mmol})$ in dry $\mathrm{THF}(28 \mathrm{~mL})$ at $0{ }^{\circ} \mathrm{C}$. The resulting mixture was stirred at $0{ }^{\circ} \mathrm{C}$ for $1 \mathrm{~h}$, then cooled to $-78{ }^{\circ} \mathrm{C}$. A solution of tropanone $3(0.14 \mathrm{~g}, 1.0 \mathrm{mmol})$ in THF $(1 \mathrm{~mL})$ was added dropwise. The resulting mixture was stirred at $-78{ }^{\circ} \mathrm{C}$ for $3 \mathrm{~h}$, and cinnamoyl cyanide $(0.20 \mathrm{~g}, 1.3 \mathrm{mmol})$ in THF $(4.0 \mathrm{~mL})$ was added. The reaction mixture was stirred at $-78^{\circ} \mathrm{C}$ for another $1 \mathrm{~h}$ and treated with a solution of silver nitrate $(0.17 \mathrm{~g}, 1.0 \mathrm{mmol})$ in THF $(0.5 \mathrm{~mL})$, water $(0.25 \mathrm{~mL})$, and acetic acid $(0.25 \mathrm{~mL})$. After warming to room temperature, the reaction mixture was treated with ammonium hydroxide solution (20\%), water, and extracted with chloroform. The extracts were dried $\left(\mathrm{MgSO}_{4}\right)$ and concentrated in vacuo. The residue was purified by flash column chromatography (silica gel, $\mathrm{CMA} 80 / \mathrm{CH}_{2} \mathrm{Cl}_{2} 1: 3$ ) to afford $7 \mathbf{a}, \mathbf{b}$ as yellow oil liquids.

(+)-Chalcostrobamine $[(+)-7 a] \cdot[\alpha]^{20}{ }_{\mathrm{D}}+166\left(\mathrm{c} 1.75, \mathrm{CHCl}_{3}\right) .{ }^{1} \mathrm{H}$ NMR $\left(300 \mathrm{MHz}, \mathrm{CDCl}_{3}\right): \delta$ $1.66(\mathrm{~m}, 1 \mathrm{H}), 1.84(\mathrm{~m}, 1 \mathrm{H}), 2.18(\mathrm{~m}, 2 \mathrm{H}), 2.30(\mathrm{~m}, 2 \mathrm{H}), 2.42(\mathrm{~s}, 3 \mathrm{H}), 2.85(\mathrm{~m}, 1 \mathrm{H}), 3.42(\mathrm{~m}$, $1 \mathrm{H}), 4.4(\mathrm{~d}, J=5.4 \mathrm{~Hz}, 1 \mathrm{H}), 6.84(\mathrm{~d}, J=15.6 \mathrm{~Hz}, 1 \mathrm{H}), 7.40(\mathrm{~m}, 3 \mathrm{H}), 7.54(\mathrm{~m}, 2 \mathrm{H}), 7.69(\mathrm{~d}, J=$ $15.6 \mathrm{~Hz}, 1 \mathrm{H}) .{ }^{13} \mathrm{C} \mathrm{NMR}\left(75 \mathrm{MHz}, \mathrm{CDCl}_{3}\right): \delta 29.11,33.38,37.11,40.82,57.66,58.84,113.06$, $117.74,127.97,128.84,129.91,135.28,140.82,172.43,197.60$.

(-)-Chalcostrobamine [(-)-7a]. The ${ }^{1} \mathrm{H}$ and ${ }^{13} \mathrm{C}$ NMR spectra were identical to those of (+)-7a. $[\alpha]^{20}-167^{\circ}\left(\mathrm{c} 1.7, \mathrm{CHCl}_{3}\right)$.

\section{General procedure for the synthesis of strobamine and analogs}

A solution of $n$-BuLi $(0.95 \mathrm{~mL}, 2.5 \mathrm{M}, 23.7 \mathrm{mmol})$ in hexane was added dropwise to a solution of $\left(R, R^{\prime}\right)$ - or $\left(S, S^{\prime}\right)$ - $\alpha, \alpha^{\prime}$-dimethyldibenzylamine $\cdot \mathrm{HCl}(0.30 \mathrm{~g}, 1.2 \mathrm{mmol})$ in anhydrous THF (28 $\mathrm{mL}$ ) at $0{ }^{\circ} \mathrm{C}$. The resulting mixture was stirred at $0{ }^{\circ} \mathrm{C}$ for $1 \mathrm{~h}$ and cooled to $-78{ }^{\circ} \mathrm{C}$. A solution of tropanone $3(0.14 \mathrm{~g}, 1.0 \mathrm{mmol})$ in THF $(1 \mathrm{~mL})$ was added dropwise. The resulting mixture was stirred at $-78^{\circ} \mathrm{C}$ for $3 \mathrm{~h}$, then the corresponding cinnamoyl cyanide $(1.3 \mathrm{mmol})$ in THF $(4.0 \mathrm{~mL})$ was added. The mixture was stirred at $-78{ }^{\circ} \mathrm{C}$ for another $1 \mathrm{~h}$, treated with a solution of silver nitrate $(0.17 \mathrm{~g}, 1.0 \mathrm{mmol})$ in THF $(0.5 \mathrm{~mL})$, water $(0.25 \mathrm{~mL})$, and acetic acid $(0.25 \mathrm{~mL})$. After warming to room temperature, the solution was extracted with chloroform. Chloroform was 
removed under vacuum, and the residue was dissolved in aqueous $2 \mathrm{~N} \mathrm{H}_{2} \mathrm{SO}_{4}$ and heated at $50{ }^{\circ} \mathrm{C}$ for $24 \mathrm{~h}$. After the solution cooled, it was adjusted to $\mathrm{pH} 11$ with conc. ammonium hydroxide and extracted with ether. The organic layers were combined, dried $\left(\mathrm{Na}_{2} \mathrm{SO}_{4}\right)$, and concentrated in vacuo. The residue was purified by flash chromatography (silica gel, EtOAc/Et $\mathrm{t}_{3} \mathrm{~N}$ 9:1) to afford $(-)$-1a-c and $(+)-2 \mathbf{a}, \mathbf{b}$ when $(R, R)-\mathbf{5}$ was used, and $(+)-\mathbf{1 a}, \mathbf{b}$ and $(-)-\mathbf{2 a}, \mathbf{b}$ when $(S, S)-\mathbf{4}$ was used as liquids.

(+)-epi-Strobamine [(+)-2a]. $100 \%$ ee by HPLC. $[\alpha]^{20}{ }_{\mathrm{D}}+91^{\circ}\left(c 0.61, \mathrm{CHCl}_{3}\right)$. IR (neat): $v 1608$, $1659 \mathrm{~cm}^{-1}$. UV (MeOH): $\lambda_{\max } 274 \mathrm{~nm} .{ }^{1} \mathrm{H}$ NMR $\left(300 \mathrm{MHz}, \mathrm{CDCl}_{3}\right): \delta 1.53(\mathrm{~m}, 1 \mathrm{H}), 1.80(\mathrm{~m}$, $1 \mathrm{H}), 1.90(\mathrm{~d}, J=18.4 \mathrm{~Hz}, 1 \mathrm{H}), 2.16(\mathrm{~m}, 2 \mathrm{H}), 2.29(\mathrm{~s}, 3 \mathrm{H}), 2.62(\mathrm{dd}, J=3.6,16.7 \mathrm{~Hz}, 1 \mathrm{H}), 2.82$ (dd, $J=5.0,18.5 \mathrm{~Hz}, 1 \mathrm{H}), 2.84(\mathrm{dd}, J=14.0,17.0 \mathrm{~Hz}, 1 \mathrm{H}), 3.36(\mathrm{~m}, 1 \mathrm{H}), 3.99(\mathrm{~d}, J=5.2 \mathrm{~Hz}$, $1 \mathrm{H}), 5.34(\mathrm{dd}, J=3.4,13.8 \mathrm{~Hz}, 1 \mathrm{H}), 7.36(\mathrm{~m}, 5 \mathrm{H}) .{ }^{13} \mathrm{C} \mathrm{NMR}\left(75 \mathrm{MHz}, \mathrm{CDCl}_{3}\right): \delta 29.16,33.46$, 34.74, 36.95, 42.33, 55.18, 58.22, 79.84, 116.73, 126.00, 128.58, 128.63, 138.29, 168.82, 189.44 . Fumarate salt: $\mathrm{mp} 131-132{ }^{\circ} \mathrm{C}$. $[\alpha]^{20}{ }_{\mathrm{D}}+77^{\circ}\left(\mathrm{c} 1.0, \mathrm{CH}_{3} \mathrm{OH}\right)$. Anal. calcd. for $\mathrm{C}_{21} \mathrm{H}_{23} \mathrm{NO}_{6} \cdot 0.5 \mathrm{H}_{2} \mathrm{O}$ : C, 63.95; H, 6.13; N, 3.55. Found: C, 63.92; H, 6.18; N, 3.51.

(-)-epi-Strobamine [(-)-2a]. $100 \%$ ee by HPLC. $[\alpha]^{20}{ }_{\mathrm{D}}-90^{\circ}\left(\mathrm{CHCl}_{3}\right)$. IR (neat): $v 1608,1659$ $\mathrm{cm}^{-1}$. UV (MeOH): $\lambda_{\max } 274 \mathrm{~nm} .{ }^{1} \mathrm{H}$ NMR and ${ }^{13} \mathrm{C}$ NMR: same as (+)-epi-strobamine (+)-2a. The fumarate salt: $\mathrm{mp} 75-82{ }^{\circ} \mathrm{C}(\mathrm{dec}) .[\alpha]^{20}{ }_{\mathrm{D}}+75^{\circ}\left(\mathrm{c} 1.2, \mathrm{CH}_{3} \mathrm{OH}\right)$. Anal. calcd. for $\mathrm{C}_{21} \mathrm{H}_{23} \mathrm{NO}_{6} \cdot 0.5 \mathrm{H}_{2} \mathrm{O}: \mathrm{C}, 63.95 ; \mathrm{H}, 6.13 ; \mathrm{N}, 3.55$. Found: $\mathrm{C}, 63.92 ; \mathrm{H}, 6.18 ; \mathrm{N}, 3.51$.

(-)-Strobamine [(-)-la]. $100 \%$ ee by HPLC. $[\alpha]^{20}{ }_{\mathrm{D}}-47^{\circ}\left(c 1.86, \mathrm{CHCl}_{3}\right)$ IR (neat): $v 1666 \mathrm{~cm}^{-1}$. $\mathrm{UV}(\mathrm{MeOH}): \lambda_{\max } 274 \mathrm{~nm} .{ }^{1} \mathrm{H} \mathrm{NMR}\left(300 \mathrm{MHz}, \mathrm{CDCl}_{3}\right): \delta 1.53(\mathrm{~m}, 1 \mathrm{H}), 1.74(\mathrm{~m}, 1 \mathrm{H}), 2.03(\mathrm{~d}, J$ $=18.6 \mathrm{~Hz}, 1 \mathrm{H}), 2.18(\mathrm{~m}, 2 \mathrm{H}), 2.37(\mathrm{~s}, 3 \mathrm{H}), 2.61(\mathrm{dd}, J=3.4,17.0 \mathrm{~Hz}, 1 \mathrm{H}), 2.81(\mathrm{dd}, J=5.1$, $18.9 \mathrm{~Hz}, 1 \mathrm{H}), 2.85(\mathrm{dd}, J=14.6,170 \mathrm{~Hz}, 1 \mathrm{H}), 3.38(\mathrm{~m}, 1 \mathrm{H}), 4.04(\mathrm{~d}, J=4.8 \mathrm{~Hz}, 1 \mathrm{H}), 5.36$ (dd, $J$ $=3.4,14.5 \mathrm{~Hz}, 1 \mathrm{H}), 7.40(\mathrm{~m}, 5 \mathrm{H}) ;{ }^{13} \mathrm{C} \mathrm{NMR}\left(75 \mathrm{MHz}, \mathrm{CDCl}_{3}\right): \delta 28.45,32.70,35.65,37.64$, $42.90,55.93,58.25,80.80,117.19,126.10,128.71,138.22$, 168.94, 189.63. Fumarate salt: $\mathrm{mp}$ 161-162 ${ }^{\circ} \mathrm{C} .[\alpha]^{20}{ }_{\mathrm{D}}-60^{\circ}\left(c \mathrm{c} 1.0, \mathrm{CH}_{3} \mathrm{OH}\right)$. Anal. calcd. for $\mathrm{C}_{17} \mathrm{H}_{20} \mathrm{ClNO}_{2} \cdot 1.5 \mathrm{H}_{2} \mathrm{O}: \mathrm{C}, 61.35 ; \mathrm{H}$, 6.97; N, 4.21. Found: C, 61.51; H, 6.90; N, 4.06.

(+)-Strobamine [(+)-la]. $100 \%$ ee by HPLC. $[\alpha]^{20}{ }_{\mathrm{D}}+48^{\circ}\left(c \quad 0.65, \mathrm{CHCl}_{3}\right)$. IR (neat): $v 1613$, $1666 \mathrm{~cm}^{-1}$. UV (MeOH): $\lambda_{\max } 274 \mathrm{~nm} .{ }^{1} \mathrm{H}$ NMR and ${ }^{13} \mathrm{C}$ NMR are the same as (-)-strobamine. Fumarate salt: $\mathrm{mp} 162-163{ }^{\circ} \mathrm{C}$. $[\alpha]^{20}{ }_{\mathrm{D}}+55^{\circ}\left(\mathrm{c} 1.1, \mathrm{CH}_{3} \mathrm{OH}\right)$. Anal. calcd. for $\mathrm{C}_{21} \mathrm{H}_{23} \mathrm{NO}_{6} \cdot 0.5 \mathrm{H}_{2} \mathrm{O}$ : C, 63.95; H, 6.13; N, 3.55. Found: C, 63.92; H, 6.18; N, 3.51.

$(+)$-epi-p-Methylstrobamine $[(+)$-epi-2b]. This compound was obtained as a solid. Recrystallization from a methanol and ether mixture gave crystals for the X-ray analysis; $100 \%$ ee by HPLC; mp $117-118{ }^{\circ} \mathrm{C}$. $[\alpha]^{20}{ }_{\mathrm{D}}+106^{\circ}\left(\mathrm{c} 1.4, \mathrm{CHCl}_{3}\right)$. IR (neat): $v 1606,1658 \mathrm{~cm}^{-1}$. UV $(\mathrm{MeOH}): \lambda_{\max } 272 \mathrm{~nm} .{ }^{1} \mathrm{H}$ NMR $\left(300 \mathrm{MHz}, \mathrm{CDCl}_{3}\right): \delta 1.56(\mathrm{~m}, 1 \mathrm{H}), 1.82(\mathrm{~m}, 1 \mathrm{H}), 1.92(\mathrm{~d}, J=$ $18.3 \mathrm{~Hz}, 1 \mathrm{H}), 2.19(\mathrm{~m}, 2 \mathrm{H}), 2.29(\mathrm{~s}, 3 \mathrm{H}), 2.37$ (s, 3H), $2.62(\mathrm{dd}, J=3.6,16.8 \mathrm{~Hz}, 1 \mathrm{H}), 2.84(\mathrm{dd}$, $J=4.8,18.6 \mathrm{~Hz}, 1 \mathrm{H}), 2.89(\mathrm{dd}, J=13.8,16.8 \mathrm{~Hz}, 1 \mathrm{H}), 3.40(\mathrm{~m}, 1 \mathrm{H}), 4.02(\mathrm{~d}, J=4.8 \mathrm{~Hz}, 1 \mathrm{H})$, $5.34(\mathrm{dd}, J=3.4,13.8 \mathrm{~Hz}, 1 \mathrm{H}), 7.21(\mathrm{~d}, J=8.0 \mathrm{~Hz}, 2 \mathrm{H}), 7.28(\mathrm{~d}, J=7.5 \mathrm{~Hz}, 2 \mathrm{H}) .{ }^{13} \mathrm{C}$ NMR $(75$ $\left.\mathrm{MHz}, \mathrm{CDCl}_{3}\right): \delta 21.10,29.17,33.50,34.87,37.10,42.30,55.22,58.27,79.90,116.64,126.15$, 129.36, 135.27, 138.64, 169.09, 189.82. Fumarate salt: $\mathrm{mp} 120-122{ }^{\circ} \mathrm{C} ;[\alpha]^{20}{ }_{\mathrm{D}}+82^{\circ}(\mathrm{c} 1.2$, 
$\mathrm{CH}_{3} \mathrm{OH}$ ). Anal. calcd. for $\mathrm{C}_{22} \mathrm{H}_{25} \mathrm{NO}_{6} \cdot 0.75 \mathrm{H}_{2} \mathrm{O}: \mathrm{C}, 63.99 ; \mathrm{H}, 6.47 ; \mathrm{N}, 3.39$. Found: $\mathrm{C}, 64.02 ; \mathrm{H}$, $6.49 ; \mathrm{N}, 3.44$.

(-)-epi-p-Methylstrobamine [(-)-epi-2b]. $100 \%$ ee by HPLC. $[\alpha]^{20}-98^{\circ}\left(c \quad 0.59, \mathrm{CHCl}_{3}\right)$. IR (neat): $v 1606,1658 \mathrm{~cm}^{-1}$. UV (MeOH): $\lambda_{\max } 272 \mathrm{~nm} .{ }^{1} \mathrm{H}$ and ${ }^{13} \mathrm{C}$ NMR: same as $(+)-e p i-1 \mathbf{b}$.

(-)-p-Methylstrobamine [(-)-1b]. $100 \%$ ee by HPLC. $[\alpha]^{20}{ }_{\mathrm{D}}-47^{\circ}\left(0.275, \mathrm{CHCl}_{3}\right)$. IR (neat): $v$ 1606, $1658 \mathrm{~cm}^{-1}$; UV (MeOH): $\lambda_{\max } 272 \mathrm{~nm} .{ }^{1} \mathrm{H}$ NMR $\left(300 \mathrm{MHz}, \mathrm{CDCl}_{3}\right): \delta 1.52(\mathrm{~m}, 1 \mathrm{H}), 1.74$ $(\mathrm{m}, 1 \mathrm{H}), 2.00(\mathrm{~d}, J=18.3 \mathrm{~Hz}, 1 \mathrm{H}), 2.16(\mathrm{~m}, 2 \mathrm{H}), 2.37(\mathrm{~s}, 6 \mathrm{H}), 2.58(\mathrm{dd}, J=3.3,17.1 \mathrm{~Hz}, 1 \mathrm{H})$, $2.80(\mathrm{dd}, J=4.8,18.6 \mathrm{~Hz}, 1 \mathrm{H}), 2.86(\mathrm{dd}, J=14.7,17.1 \mathrm{~Hz}, 1 \mathrm{H}), 3.68(\mathrm{~m}, 1 \mathrm{H}), 4.04(\mathrm{~d}, J=4.5$ $\mathrm{Hz}, 1 \mathrm{H}), 5.32(\mathrm{dd}, \mathrm{J}=3.3,14.7 \mathrm{~Hz}, 1 \mathrm{H}), 7.21(\mathrm{~d}, J=8.1 \mathrm{~Hz}, 2 \mathrm{H}), 7.28(\mathrm{~d}, \mathrm{~J}=8.1 \mathrm{~Hz}, 2 \mathrm{H}) .{ }^{13} \mathrm{C}$ NMR $\left(75 \mathrm{MHz}, \mathrm{CDCl}_{3}\right): \delta 21.11,28.42,32.68,35.67,37.67,42.78,55.91,58.21,80.75,117.08$, $126.16,129.35,135.18,138.67,169.04,189.85$. The fumarate salt had $\mathrm{mp} 77-85{ }^{\circ} \mathrm{C}(\mathrm{dec}) ;[\alpha]^{20}{ }_{\mathrm{D}}$ $-52^{\circ}$ (c 1.4, $\left.\mathrm{CH}_{3} \mathrm{OH}\right)$. Anal. calcd. for $\mathrm{C}_{22} \mathrm{H}_{25} \mathrm{NO}_{6} \cdot 0.5 \mathrm{H}_{2} \mathrm{O}: \mathrm{C}, 64.69 ; \mathrm{H}, 6.42 ; \mathrm{N}, 3.43$. Found: $\mathrm{C}$, 64.70; H, 5.56; N, 3.48.

(+)-p-Methylstrobamine [(+)-1b]. $100 \%$ ee by HPLC. $[\alpha]^{20}{ }_{\mathrm{D}}+49^{\circ}\left(0.275, \mathrm{CHCl}_{3}\right)$. IR (neat): $\tilde{v}$ $1606,1658 \mathrm{~cm}^{-1}$. UV (MeOH): $\lambda_{\max } 272 \mathrm{~nm} .{ }^{1} \mathrm{H}$ and ${ }^{13} \mathrm{C}$ NMR: same as $(-)-\mathbf{1 b}$.

\section{X-Ray crystal structure determination of [(+)-epi-2b]}

Single-crystal X-ray diffraction data on (+)-epi-methylstrobamine $(+)$-epi-2b was collected at $293^{\circ} \mathrm{K}$ using $\mathrm{CuKa}$ radiation produced by a Bruker Micro-STAR rotating anode equipped with Helios optics and a Bruker Platinum-135 CCD area detector. Crystals were prepared for data by mounting on the end of a thin glass rod using an acrylic adhesive. Corrections were applied for Lorentz, polarization, and absorption effects. The crystal was orthorhombic in space group P21212 with unit cell dimensions $\mathrm{a}=11.2517(6) \AA, \mathrm{b}=21.5455(12) \AA, \mathrm{c}=6.5032(4) \AA$. Data were $97.2 \%$ complete to $68.12^{\circ} \mathrm{q}$ (approximately $0.83 \AA$ ), with an average redundancy of 4.92 . The structure was solved by direct methods and refined by full-matrix least squares on F2 values using the programs found in the SHELXTL suite (Bruker, SHELXTL v6.10, 2000, Bruker AXS Inc., Madison, WI). Parameters refined included atomic coordinates and anisotropic thermal parameters for all non-hydrogen atoms. Hydrogen atoms on carbons were included using a riding model (coordinate shifts of $\mathrm{C}$ applied to $\mathrm{H}$ atoms) with $\mathrm{C}-\mathrm{H}$ distance set at $0.96 \AA$. The absolute configuration was evaluated using likelihood methods in PLATON. ${ }^{6,7}$ Based on the analysis of 1077 Bijvoet pairs (90\% coverage), this analysis indicated that the absolute structure had been correctly assigned. The method calculated that the probability that the structure is inverted is smaller than $1 \times 10-34$. Atomic coordinates for compound (+)-epi-2b have been deposited with the Cambridge Crystallographic Data Centre (deposition number 776942). Copies of the data can be obtained, free of charge, on application to CCDC, 12 Union Road, Cambridge, CB2 1EZ, UK [fax: +44(0)-1223-336033 or e-mail: deposit@ccdc.cam.ac.uk]. 


\section{Acknowledgements}

This work was supported in part by the National Institute on Drug Abuse grant number DA05477. Support for the X-ray crystallographic studies was provided by the National Institute on Drug Abuse contract Y1-DA6002.

\section{References}

1. Lounasmaa, M.; Pusset, J.; Sevenet, T. Phytochemistry 1980, 19, 949.

2. Lounasmaa, M.; Pusset, J.; Sevenet, T. Phytochemistry 1980, 19, 953.

3. Lounasmaa, M.; Holmberg, C.; Langenskiold, T. J. Nat. Prod. 1983, 46, 429.

4. Majewski, M.; Lazny, R. J. Org. Chem. 1995, 60, 5825.

5. Olah, G. A.; Arvanaghi, M.; Prakash, G. K. S. Synthesis 1983, 636.

6. Spek, A. L. PLATON (ver. 310310), A Multipurpose Crystallographic Tool, Utrecht University, Utrecht, The Netherlands, 2010.

7. Hooft, R. W. W.; Straver, L. H.; Spek, A. L. J. Appl. Crystallogr. 2008, 41, 96. 